\title{
PSYCHOLOGICAL WELL-BEING IN PATIENTS WHO SUFFER FROM BREAST CANCER
}

\author{
Iva Binic, Olivera Zikic, Gordana Nikolic, Jelena \\ Kostic, Ana Cvetanovic, Jovana Antonijevic, Jelena \\ Stojanov
}

Medical Faculty, University of Nis, Serbia

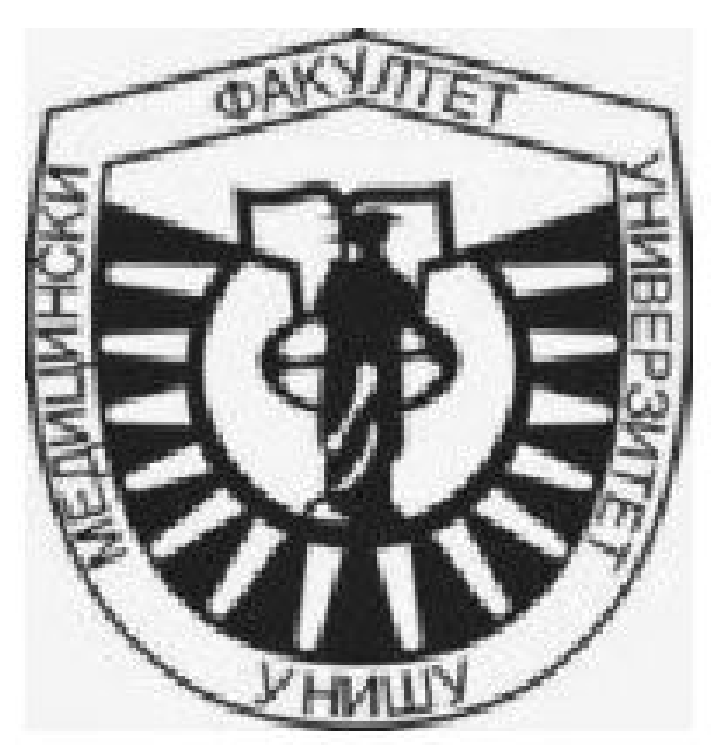

BACKGROUND: Breast cancer patients suffer from several negative consequences after treatment and these include pain, fatigue, sexual problems, appearance and body image concerns, with psychological dysfunction. This could affect the patients psychological well-being. According to the literature, almost $50 \%$ of cancer patients suffer from psychiatric disorders, and anxiety and depression are considered to be the most important psychopathological comorbidities.

OBJECTIVES: The aim was to examine the psychological well-being among patients who suffer from breast cancer.

METHODS: In total, 95 patients with breast cancer were enrolled in the study with completed surgical treatment and the actual administration of adjuvant therapy. The data were obtained through interviews with patients and through a review of their medical history. We used Hospital anxiety and depression inventory (HADS) to assess the intensity of anxiety and depression in somatic patients, 15-item Flanagan's Quality of Life Scale (QOLS) modified by adding a 16th item on independence to measure quality of life, Holmes and Rahe Stress Scale to assess the level of stress in the past year.

RESULTS: The results of our study showed a negative correlation between the quality of life and the anxiety score ( $p=$ $0.022)$, depression $(p<0.001)$ and total stress $(p=0.011)$. Better psychological well being was observed in those patients who had lower values of depression, stress and anxiety.

CONCLUSION: A breast cancer diagnosis can severely impair a woman's psychological functioning, and there is a need for psychosocial support programs and psychological screening and consultations for patients diagnosed with breast cancer.

Key words: breast cancer, well being, psychological 